\title{
Enhanced Expression of NR2B Subunits of NMDA Receptors in the Inherited Glaucomatous DBA/2J Mouse Retina
}

\author{
Ling-Dan Dong, ${ }^{1,2,3}$ Jie Chen, ${ }^{1,2,3}$ Fang Li, ${ }^{1,2,3}$ Feng Gao, ${ }^{1,3,4}$ Jihong Wu, ${ }^{1,3,4}$ \\ Yanying Miao, ${ }^{1,2,3}$ and Zhongfeng Wang ${ }^{1,2,3,4}$ \\ ${ }^{1}$ Institutes of Brain Science, Fudan University, 138 Yixueyuan Road, Shanghai 200032, China \\ ${ }^{2}$ Institute of Neurobiology, Fudan University, 138 Yixueyuan Road, Shanghai 200032, China \\ ${ }^{3}$ State Key Laboratory of Medical Neurobiology, Fudan University, 138 Yixueyuan Road, Shanghai 200032, China \\ ${ }^{4}$ Eye \& ENT Hospital, Fudan University, 83 Fenyang Road, Shanghai 200031, China \\ Correspondence should be addressed to Yanying Miao; yymiao@fudan.edu.cn and Zhongfeng Wang; zfwang@fudan.edu.cn
}

Received 8 July 2013; Accepted 14 August 2013

Academic Editor: Sheng Tian Li

Copyright (c) 2013 Ling-Dan Dong et al. This is an open access article distributed under the Creative Commons Attribution License, which permits unrestricted use, distribution, and reproduction in any medium, provided the original work is properly cited.

\begin{abstract}
DBA/2J mouse has been used as a model for spontaneous secondary glaucoma. Here, we investigated changes in expression of NMDA receptor (NMDAR) subunits and Cdk5/p35/NMDAR signaling in retinas of DBA/2J mice using Western blot technique. The protein levels of NR1 and NR2A subunits in retinas of DBA/2J mice at all ages (6-12 months) were not different from those in age-matched C57BL/6 mice. In contrast, the protein levels of NR2B subunits, in addition to age-dependent change, significantly increased with elevated intraocular pressure (IOP) in DBA/2J mice at 6 and 9 months as compared with age-matched controls. Moreover, expression of $\mathrm{Cdk} 5, \mathrm{p} 35$ and ratio of $\mathrm{p}-\mathrm{NR} 2 \mathrm{~A}^{\mathrm{S1232}} / \mathrm{NR} 2 \mathrm{~A}$ progressively increased with time in both strains, suggestive of activated Cdk5/p35 signaling pathway. However, the changes in these proteins were in the same levels in both strain mice, except a significant increase of $\mathrm{p} 35$ proteins at 6 months in DBA/2J mice. Meanwhile, the protein levels of Brn-3a, a retinal ganglion cell (RGC) maker, remarkably decreased at 9-12 months in DBA/2J mice, which was in parallel with the changes of NR2B expression. Our results suggest that elevated IOP-induced increase in expression of NR2B subunits of NMDARs may be involved in RGC degeneration of DBA/2J mice.
\end{abstract}

\section{Introduction}

Glaucoma, the second leading cause of blindness worldwide, is a neurodegenerative disease characterized by apoptotic death of retinal ganglion cells (RGCs) and progressive visual field loss [1,2], which is often associated with high intraocular pressure (IOP). Whilst the mechanisms of RGC death in glaucoma still remain a mystery, glutamate excitotoxicity triggered by overactivation of the $\mathrm{N}$-methyl-D-aspartate receptors (NMDARs) may be a potential risk factor for retinal malfunction in glaucoma [3-5]. Indeed, delivery of NMDA channel blockers has been shown to effectively reduce RGC apoptosis in experimental rat glaucoma models [3, 6-8]. Our recent work also showed that cyclin-dependent kinase 5 (Cdk5)/p35-induced elevation of phosphorylated NR2A subunit of NMDARs at S1232 site (p-NR2A ${ }^{\text {S1232 }}$ ) may contribute to RGC apoptotic death in experimental glaucomatous rats [9].
DBA/2J mouse is a spontaneous model of glaucomatous neurodegeneration, which develops a progressive form of pigmentary angle-closure glaucoma [10-13]. In these mice, IOPs become elevated by 6 months of age, and continued intraocular hypertension results in progressive RGC degeneration [12-17]. This is similar to what is observed in primary open angle glaucoma, which makes the DBA/2J mice represent a useful model to study mechanisms of RGC death of human glaucoma [1, 11]. Previous studies have demonstrated that NMDAR antagonist memantine treatment significantly increased RGC survival in DBA/2J mice by inhibiting mitochondrial OPA1 and cytochrome $\mathrm{c}$ release, decreasing Bax gene expression and increasing $\mathrm{Bcl}-2$ gene expression, suggesting that overactivation of NMDARs in the glaucomatous DBA/2J retina may lead to a distinct mitochondria-mediated RGC death pathway $[15,18]$. However, whether expression of NMDARs is changed in DBA/2J retina is largely unknown. The only evidence for this is that 
no changes in expression of NMDAR subunits were seen in DBA/2J mice by immunohistochemistry in retinal slices [19]. In the present work, we aimed to examine changes in expression of NMDAR subunits and Cdk5/p35/NMDAR signaling in retinas of $\mathrm{DBA} / 2 \mathrm{~J}$ mice at various ages $(3,6,9$, and 12 months) using Western blot technique.

\section{Material and Methods}

2.1. Animals. Male DBA/2J mice, obtained from The Jackson Laboratory (Bar Harbor, ME, USA), and age-matched C57BL/6 mice, obtained from SLAC Laboratory Animal Co. Ltd (Shanghai, China), were housed on a $12 \mathrm{~h}$ light/dark schedule, with standard food and water provided ad libitum. All experimental procedures described here were carried out in accordance with the National Institutes of Health (NIH) guidelines for the Care and Use of Laboratory Animals and the guidelines of Fudan University on the ethical use of animals. During this study, all possible efforts were made to minimize the number of animals used and their suffering.

2.2. IOP Measurement. IOPs of both eyes in DBA/2J mice and age-matched C57BL/6J mice were measured using a handheld digital tonometer (TonoLab, TioLat, Finland) under general and local anesthesia as described previously $[9,20]$. The average value of five consecutive measurements with a deviation of less than $5 \%$ was accepted. All measurements were performed in the morning to avoid possible circadian difference.

2.3. Western Blot Analysis. Western blot analysis was conducted as previously described with some modifications [9, 20]. DBA/2J mice or age-matched C57BL/6 mice in different ages $(3,6,9$, and 12 months) were deeply anesthetized with $25 \%$ urethane $(1.25 \mathrm{~g} / \mathrm{kg}$, i.p.). The retinas were removed quickly and snap frozen in liquid nitrogen and then stored at $-80^{\circ} \mathrm{C}$ for further use. Retinas were homogenized in RIPA lysis buffer (50 mM Tris-Cl, $150 \mathrm{mM} \mathrm{NaCl,} \mathrm{1 \%} \mathrm{Triton} \mathrm{X-100,}$ $0.1 \%$ aprotinin, $1 \mathrm{mM}$ phenylmethylsulfonyl fluoride, $1 \mathrm{mM}$ sodium orthovanadate, and $25 \mathrm{mM}$ sodium fluoride, $\mathrm{pH}$ 7.4), supplemented with protease and phosphatase inhibitor cocktail (Roche, Mannheim, Germany). The concentration of total proteins was measured using a standard bicinchoninic acid (BCA) assay kit (Pierce Biotechnology, IL, USA). The extracted protein samples $(20 \mu \mathrm{g}, 15 \mu \mathrm{L}$ in volume) were resolved by $8 \%$ or $15 \%$ SDS-PAGE gel and electroblotted onto PVDF membranes (Immobilon-P, Millipore, Billerica, MA, USA) using Mini-PROTEAN 3 Electrophoresis System and Mini Trans-Blot Electrophoretic Transfer System (BioRad, Hercules, CA, USA). After blocking in 5\% nonfat milk at room temperature for $1 \mathrm{~h}$, the membranes were incubated overnight at $4^{\circ} \mathrm{C}$ with primary antibodies. The primary antibodies used in the present work include antiNR1 (\#05432, 1:500, Millipore, Billerica, MA, USA), anti-pNR2A ${ }^{\text {S1232 }}$ (\#2056, 1:500, Tocris Bioscience, MO, USA), antiNR2A (\#320600, 1:500, Invitrogen, Carlsbad, CA, USA), anti-Brn-3a (sc-8429, 1: 1000, Santa Cluz, Biotechnology, CA, USA), anti-Cdk5 (\#20502, clone DC17, 1:1000, Millipore),



$\mathrm{C} 57 \mathrm{BL} / 6$

$\mathrm{DBA} / 2 \mathrm{~J}$

FIGURE 1: Changes of IOPs in C57BL/6 and DBA/2J mice at various ages. Bar chart showing changes of the average IOPs in C57BL/ 6 and DBA/2J mice at ages of $3(n=24), 6(n=18), 9(n=12)$ and 12 $(n=6)$ months. All data are presented as mean \pm S.E.M. ${ }^{* * *} P<$ 0.001 versus 3 -month-old C57BL/6 mice; ${ }^{\# \# \# ~} P<0.001$ versus agematched C57BL/6 mice.

anti-NR2B (\#06600, 1:500, Millipore), anti-p35/p25 (sc820, 1:1000; Santa Cruz Biotechnology), $\beta$-actin antibody (A5316, 1:2000, Sigma, Saint Louis, MO, USA), and antiGAPDH (1:1000, Cell Signal Technology, MA, USA). After washing in Tris-buffered saline-Tween 20, the membranes were incubated with horseradish-peroxidase-(HRP-) conjugated donkey anti-mouse or donkey anti-rabbit secondary antibody (Thermo Scientific, Rockford, IL, USA) at a 1:2500 dilution for $1 \mathrm{~h}$ at room temperature. The blots were then incubated with chemifluorescent reagent ECL (Thermo Scientific, Rockford, IL, USA) and exposed to X-ray film in the dark. The experiments were performed in triplicate, and the protein bands were quantitatively analyzed with NIH Image J Analysis software.

2.4. Statistical Analysis. All data are presented as mean \pm S.E.M. Statistical analysis was performed by using the Graphpad Prism software (version 5.0; Graphpad Software, San Diego, CA, USA). A one-way or two-way analysis of variance (ANOVA) with nonparametric test (Kruskal-Wallis test), Bonferroni's post hoc test (multiple comparisons), and Mann-Whitney test (comparisons between two groups) was used as appropriate. A value of $P<0.05$ was considered significant.

\section{Results}

3.1. Changes in Expression of NMDAR Subunits in $D B A / 2 J$ Mice at Various Ages. We first monitored changes in IOPs of DBA/2J mice with time. As shown in Figure 1, the average IOP of DBA/2J mice was $14.8 \pm 0.5 \mathrm{~mm} \mathrm{Hg}(n=24)$ at age of 3 months, which was comparable to that of age-matched C57BL/ 6 control mice $(13.6 \pm 0.6 \mathrm{~mm} \mathrm{Hg})(n=24)$. The average IOP of control mice kept this level at ages of 6, 9, and 12 months, while that of DBA/2J mice significantly increased to $18.5 \pm 0.9 \mathrm{~mm} \mathrm{Hg}(n=18, P<0.001)$ and $19.9 \pm 1.0 \mathrm{~mm} \mathrm{Hg}$ ( $n=12, P<0.001)$ at ages of 6 and 9 months, respectively, 
and then declined to $15.6 \pm 0.8 \mathrm{~mm} \mathrm{Hg}(n=6, P>0.05)$ at age of 12 months (Figure 1). In the present study, all of the DBA/2J mice showed progressive elevated IOPs. Therefore, our data included all animals.

Expression of NR1 subunit of NMDARs was unchanged at the age of 6 months both in DBA/2J and C57BL/6 mice $(101.0 \pm 13.2 \%$ and $95.0 \pm 8.1 \%$ of that at age of 3 months (control), $n=6, P>0.05$ ) (Figures 2(a) and 2(b)). While expression of this protein increased to $133.4 \pm 11.2 \%$ of control at age of 9 months in DBA/2J mice $(n=6, P<0.01)$, following by a decline to $90.1 \pm 19.2 \%$ of control at age of 12 months, it was not different from that of age-matched C57BL/6 mice $(128.2 \pm 17.1 \%$ of control, $P<0.01$ for 9 months; $110.2 \pm 12.1 \%$ of control, $P>0.05$ for 12 months, resp.) (Figures 2(a) and 2(b)). Expression of NR2A subunit was elevated at ages of 9 and 12 months in DBA/2J mice $(135.3 \pm 14.1 \%$ and $148.2 \pm 17.2 \%$ of control, resp., $n=6$, all $P<0.001)$, which was comparable to age-matched C57BL/6 mice $(125.3 \pm 16.1 \%(n=6, P<0.01)$ and $140.0 \pm 12.1 \%$ of control ( $n=6, P<0.001)$, resp.) (Figures 2(a) and 2(c)). Meanwhile, the ratio of $\mathrm{p}-\mathrm{NR} 2 \mathrm{~A}^{\mathrm{S} 1232} / \mathrm{NR} 2 \mathrm{~A}$ showed a remarkable increase at ages of 6 and 9 months in DBA/2J mice $(145.4 \pm 16.0 \%(n=6, P<0.001)$ and $128.0 \pm 17.0 \%$ of control ( $n=6, P<0.001)$, resp.), and at the age of 6 months in age-matched C57BL/6 mice (134.5 $\pm 19.0 \%$ of control, $n=6, P<0.01$ ) (Figures $2(\mathrm{a})$ and $2(\mathrm{~d})$ ). However, the ratio

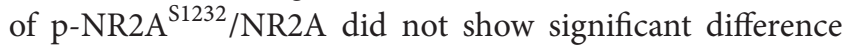
between DBA/2J and age-matched C57BL/6 mice at all ages. Expression of NR2B subunit was quite different (Figure 2(a)). The average level of NR2B proteins increased to $166.0 \pm 19.1 \%$ $(n=6, P<0.05), 446.3 \pm 15.0 \%(n=6, P<0.001)$, and $541.4 \pm 19.3 \%$ of control $(n=6, P<0.001)$ in C57BL/ 6 mice at ages of 6, 9, and 12 months. Expression of NR2B proteins in DBA/2J mice showed a dramatic increase, with average protein levels being $287.0 \pm 29.4 \%(n=6, P<0.001$ versus control and $P<0.001$ versus age-matched C57BL/ 6 mice), $517.3 \pm 62.0 \%(n=6, P<0.001$ versus control and $P<0.001$ versus age-matched C57BL/ 6 mice) and $540.4 \pm 58.1 \%$ of control $(n=6, P<0.001)$ at ages of 6,9 , and 12 months, respectively (Figures $2(\mathrm{a})$ and $2(\mathrm{e})$ ).

\subsection{Changes in Expression of Cdk5 and $p 35$ in DBA/2J Mice} at Various Ages. Since the expression of $\mathrm{p}-\mathrm{NR} 2 \mathrm{~A}^{\mathrm{S} 1232}$ was at high level (Figure 2), we examined changes of its activator Cdk5 and coactivator p35/p25 [9]. As shown in Figure 3(a), the protein level of Cdk5 progressively increased with time in DBA/2J mice, with the average protein density being $148.1 \pm$ $17.0 \%(n=6, P<0.001), 169.7 \pm 18.1 \%(n=6, P<0.001)$, and $165.8 \pm 18.0 \%$ of control $(n=6, P<0.001)$ at ages of 6,9 , and 12 months, respectively (Figure 3(b)). However, there was no significant difference between DBA/2J mice and age-matched C57BL/6 mice at all ages (Figures 3(a) and $3(\mathrm{~b})$ ). Similarly, the $\mathrm{p} 35$ protein level gradually increased to $168.7 \pm 21.1 \%(n=6, P<0.01), 229.7 \pm 26.3 \%$ of control $(n=6, P<0.001)$ in DBA/2J mice at ages of 6 and 9 months, and then slightly declined to $191.3 \pm 25.1 \%$ of control $(n=6, P<0.001)$ at the age of 12 months (Figures 3(a) and 3(c)). Except at age of 6 months $(n=6, P<0.001$ versus age-matched C57BL/6 mice), the p35 protein levels at all other ages were comparable to those of age-matched C57BL/6 mice (Figures 3(a) and 3(c)). At the same time, p25 protein, a truncated form of $\mathrm{p} 35$, was not detected both in $\mathrm{DBA} / 2 \mathrm{~J}$ and $\mathrm{C} 57 \mathrm{BL} / 6$ mice.

3.3. Changes in Expression of Brn-3a in DBA/2J Mice at Various Ages. We finally examined changes in expression of Brn3a, a RGC marker, to evaluate RGC damage. Figure 4(a) shows representative Western blot results. The protein level of Brn-3a in DBA/2J mice was higher than that of control mice at ages of 3 and 6 months $(127.6 \pm 4.8 \%$ and $129.9 \pm 5.4 \%$ of control, $n=4$, all $P<0.05)$, whereas it decreased to $83.3 \pm$ $2.7 \%(n=4, P<0.05$ versus control and age-matched C57BL/6 mice; $P<0.01$ versus DBA/2J mice at age of 3 months) and $86.7 \pm 1.8 \%$ of control $(n=4, P<0.05$ versus control and age-matched C57BL/ 6 mice; $P<0.01$ versus $\mathrm{DBA} / 2 \mathrm{~J}$ mice at age of 3 months) at ages of 9 and 12 months (Figure 4(b)).

\section{Discussion}

In the present study, we found that expression of NR2B subunits of NMDARs in retinas of the DBA/2J mice was gradually enhanced with time. Progressive elevated IOPinduced increase in NR2B expression may be associated with RGC degeneration in this glaucomatous model.

Glutamate excitotoxicity has been implicated in glaucomatous RGC death, which is primarily mediated by NMDARs [3, 21]. As a spontaneous glaucomatous model, overactivation of NMDARs by glutamate may be also involved in RGC degeneration of DBA/2J mice. This was supported by the experimental results. First, it was reported that vitreal glutamate content measured with HPLC in DBA/2J mice was higher than that of age-matched controls, and glutamate transporters GLAST and GLT-1v expression in DBA/2J mice showed a decrease by Western blotting [19]. Secondly, blocking NMDARs by memantine inhibited RGC apoptosis and increased RGC survival in DBA/2J mice [15, 18]. It should be noted that time-dependent loss of RGCs in DBA/2J mice started at 6 months of age [16], in parallel with progressive elevation of IOPs and changes of glutamate transporters and glutamate concentration [19].

Previous work has demonstrated that expression of both NMDAR and AMPA receptor (AMPAR) subunits did not show age-dependent change in the retinas of DBA/2J mice by immunohistochemistry [19]. Consistent with this, our results revealed that there was no significant change in expression of NR1 subunits in the retina of DBA/2J mice as compared with age-matched C57BL/6 mice by Western blot analysis. In human glaucomatous eyes, however, NR1 levels showed a decrease [22]. Even though there was no difference between DBA/2J mice and age-matched C57BL/6 mice in the expression of NR2A subunits at all ages, age-dependent increase in NR2A expression was observed at 9 and 12 months in both DBA/2J and C57BL/6 mice (Figure 2(c)). A major finding in this work is that, in addition to agedependent change, expression of NR2B subunits significantly 


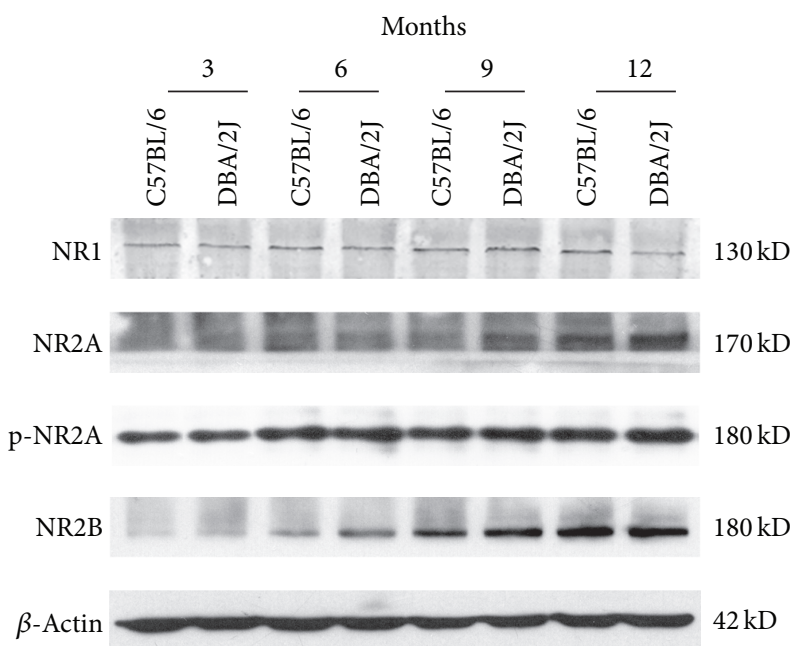

(a)

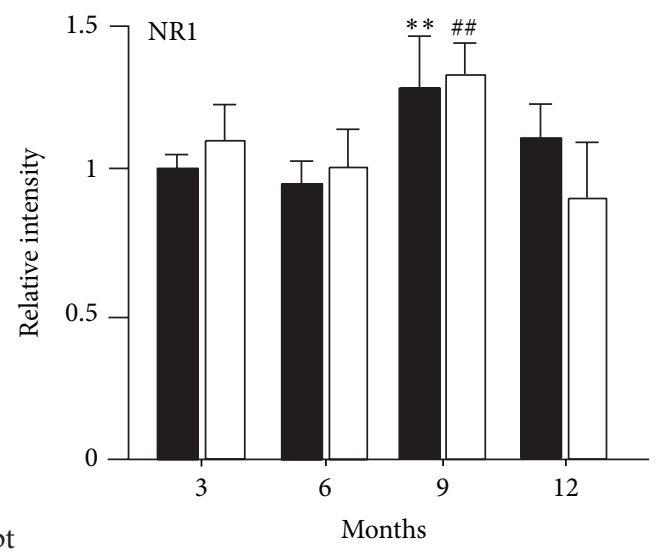

(b)



C57BL/6 DBA/2J

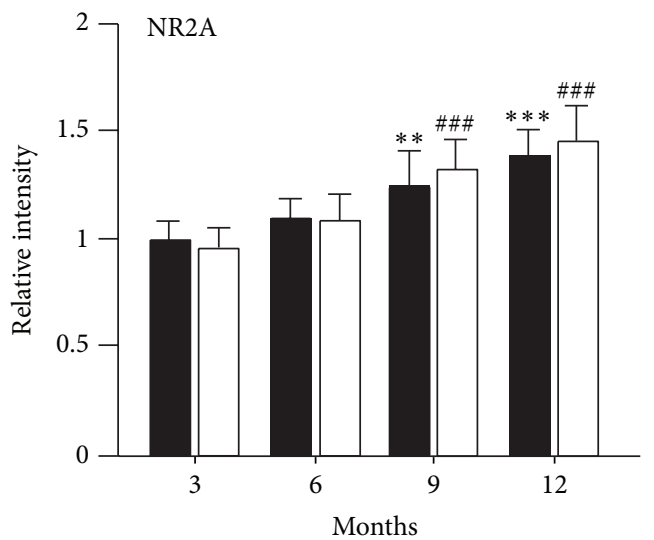

(c)



(e)

FIGURE 2: Changes in expression of NMDA receptor subunits of DBA/2J mice at various ages. (a) Representative immunoblots showing the changes of NR1, NR2A, p-NR2A ${ }^{\text {S1232 }}$, NR2B levels in DBA/2J and C57BL/6 mice at ages of 3, 6, 9, and 12 months. (b)-(e) Bar chart showing the average densitometric quantification of immunoreactive bands of NR1 (b), NR2A (c), p-NR2A $A^{\mathrm{S} 1232} / \mathrm{NR} 2 \mathrm{~A}(\mathrm{~d})$, and NR2B (e) in DBA/2J and C57BL/6 mice at ages of 3, 6, 9, and 12 months, respectively. ${ }^{*} P<0.05,{ }^{* *} P<0.01$ and ${ }^{* * *} P<0.001$ versus 3-month-old C57BL/6 mice; ${ }^{\# \#} P<0.01$ and ${ }^{\# \# \#} P<0.001$ versus 3 -month-old DBA/2J; ${ }^{\& \& \&} P<0.001$ versus age-matched C57BL/6 mice. 


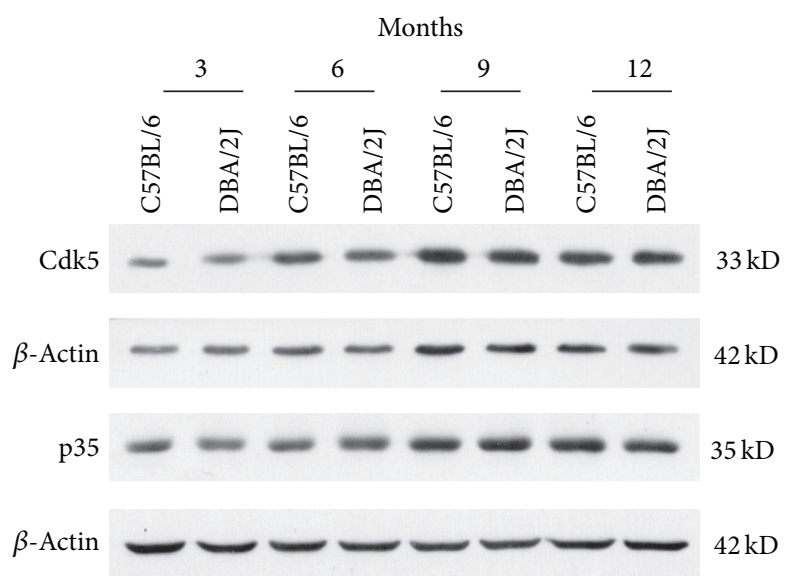

(a)

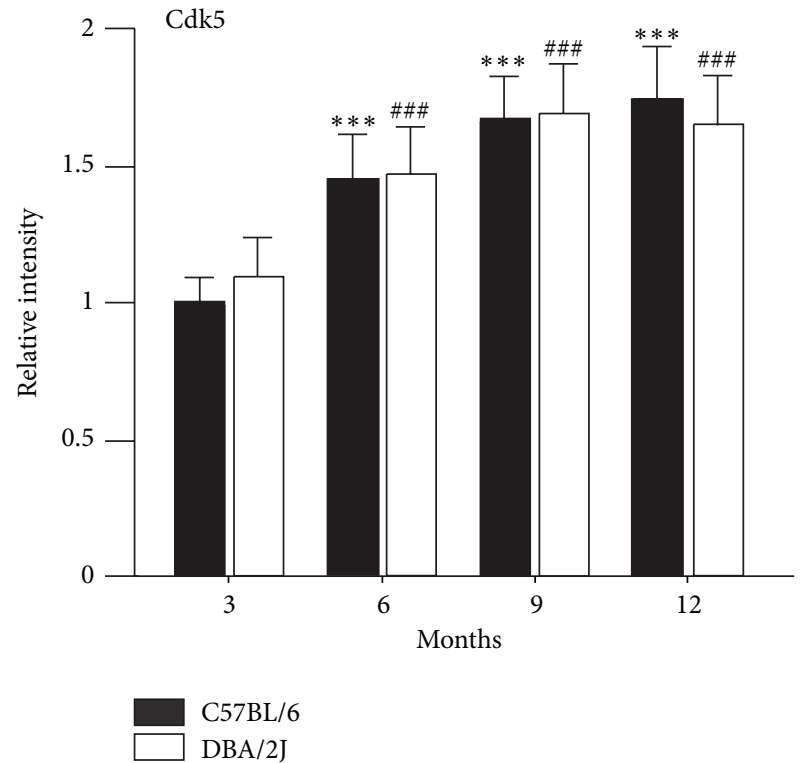

(b)

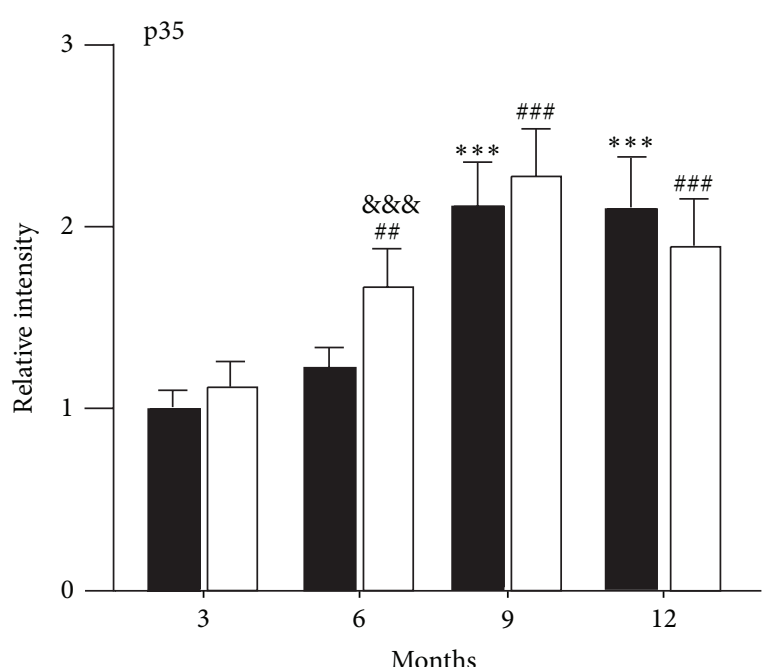

C57BL/6 $\mathrm{DBA} / 2 \mathrm{~J}$

(c)

FIGURE 3: Changes in expression of Cdk5, $p 35$ of DBA/2J mice at various ages. (a) Representative immunoblots showing the changes of Cdk5 and p35 levels in DBA/2J and C57BL/6 mice at ages of 3, 6, 9, and 12 months. ((b), (c)) Bar chart showing the average densitometric quantification of immunoreactive bands of Cdk5 (b) and p35 (c) in DBA/2J and C57BL/6 mice at ages of 3, 6, 9, and 12 months, respectively. ${ }^{* * *} P<0.001$ versus 3-month-old C57BL/6 mice; ${ }^{\# \#} P<0.01$ and ${ }^{\# \# \#} P<0.001$ versus 3-month-old DBA/2J; ${ }^{\text {\&\&\& }} P<0.001$ versus age-matched C57BL/6 mice.

increased accompanyied with elevated IOPs in DBA/2J mice (Figure 2(e)). It was recently reported that NMDARs located at the synapse stimulate cell survival pathways, while extrasynaptic receptors signal for cell death [23-26]. NR2B subunits are commonly associated with extrasynaptic locations at the synapse, thus involving in neurologic diseases and some neurodegenerative disorders [24-30]. In the retina, it was reported that NR2A and NR2B subunits were expressed predominantly synaptically and perisynaptically respectively [31]. Therefore, we deduced that excessive glutamate may stimulate the overexpressed extrasynaptic NR2B subunits in $\mathrm{DBA} / 2 \mathrm{~J}$ mice, thus triggering cell death signals. Consistently, Brn-3a expression was decreased following the changes of NR2B expression (Figure 4), indicating a correlation between overexpression of NR2B and RGC damage. Although Brn-3a expression was highly related to increase of NR2B expression, it is noteworthy that overactivation of NMDARs is not a sole factor for RGC degeneration in DBA/2J mice. Moreover, Brn3a expression in DBA/2J mice was higher than control at ages of 3 and 6 months. It is possible that as a transcript factor, Brn-3a protein levels could not accurately reflect the number of RGCs, which is worthwhile to be further explored.

Our previous work found that Cdk5/p35 signaling pathway was activated in a rat experimental glaucoma model, and the activated $\mathrm{Cdk} 5 / \mathrm{p} 35$ signaling in turn induced an elevation of p-NR2A ${ }^{\mathrm{S} 1232}$ expression, which contributed to rat RGC apoptotic death [9]. Indeed, we found that the expression of Cdk5, p35 and the ratio of $\mathrm{p}-\mathrm{NR} 2 \mathrm{~A}^{\mathrm{S} 1232} / \mathrm{NR} 2 \mathrm{~A}$ progressively 


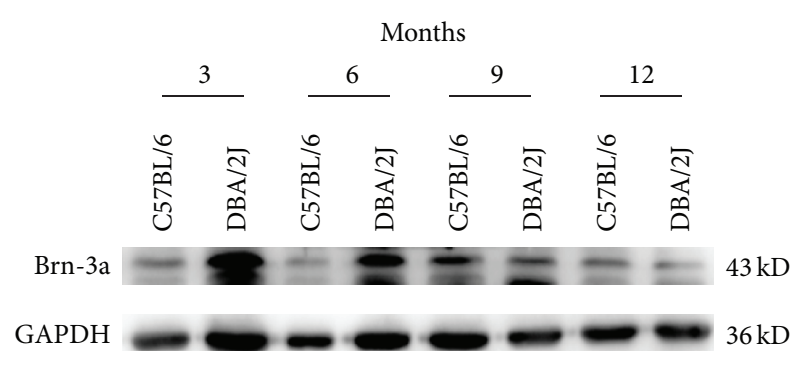

(a)



C57BL/6

$\mathrm{DBA} / 2 \mathrm{~J}$

(b)

FIGURE 4: Changes in expression of Brn-3a of DBA/2J mice at various ages. (a) Representative immunoblots showing the changes of Brn-3a levels in DBA/2J and C57BL/6 mice at ages of 3, 6, 9, and 12 months. (b) Bar chart showing the average densitometric quantification of immunoreactive bands of Brn-3a in DBA/2J and C57BL/6 mice at ages of 3, 6, 9, and 12 months, respectively. ${ }^{*} P<0.05$ versus 3 month-old C57BL/6 mice; ${ }^{\# \#} P<0.01$ versus 3 -month-old DBA/2J mice; ${ }^{\&} P<0.05$ versus age-matched C57BL/6 mice.

increased with time in retinas of DBA/2J mice, suggestive of activated Cdk5/p35 signaling pathway. However, the changes in these protein levels were comparable to those in agematched C57BL/6 mice, except a significant increase in p35 expression of DBA/2J mice at age of 6 months. These results suggest that progressive moderate elevation of IOP in DBA/2J mice is unlikely a primary factor for activation of Cdk5/p35/NMDAR signaling pathway. Aging plays an important role in activating this signaling pathway. On the other hand, activated Cdk5/p35 signaling pathway may modulate NR2B subunits and increase their expression in DBA/2J mice since it was reported that Cdk5 may indirectly regulate NR2B [32].

In conclusion, our results suggest that NMDARs may be involved in RGC degeneration of DBA/2J mice through two pathways: IOP elevation-induced increase in expression of NR2B subunits and age-dependent activation of Cdk5/ p35/NMDAR signaling pathway.

\section{Conflict of Interests}

There is no conflict of interests.

\section{Acknowledgments}

This work was supported by grants from the National Program of Basic Research sponsored by the Ministry of Science and Technology of China (2013CB835101; 2011CB504602), the Natural Science Foundation of China (30930034; 31070966; 31271173), and the Key Research Program of Science and Technology Commissions of Shanghai Municipality, China (11JC1401200).

\section{References}

[1] L. Guo, S. E. Moss, R. A. Alexander, R. R. Ali, F. W. Fitzke, and M. F. Cordeiro, "Retinal ganglion cell apoptosis in glaucoma is related to intraocular pressure and IOP-induced effects on extracellular matrix," Investigative Ophthalmology \& Visual Science, vol. 46, no. 1, pp. 175-182, 2005.

[2] R. A. Hitchings, "Selective ganglion cell death in glaucoma," British Journal of Ophthalmology, vol. 84, no. 7, pp. 678-679, 2000.

[3] L. Guo, T. E. Salt, A. Maass et al., "Assessment of neuroprotective effects of glutamate modulation on glaucoma-related retinal ganglion cell apoptosis in vivo," Investigative Ophthalmology \& Visual Science, vol. 47, no. 2, pp. 626-633, 2006.

[4] L. A. Levin, "Retinal ganglion cells and neuroprotection for glaucoma," Survey of Ophthalmology, vol. 48, no. 2, supplement, pp. S21-S24, 2003.

[5] M. Seki and S. A. Lipton, "Targeting excitotoxic/free radical signaling pathways for therapeutic intervention in glaucoma," Progress in Brain Research, vol. 173, pp. 495-510, 2008.

[6] J. I. Calzada, B. E. Jones, P. A. Netland, and D. A. Johnson, "Glutamate-induced excitotoxicity in retina: neuroprotection with receptor antagonist, dextromethorphan, but not with calcium channel blockers," Neurochemical Research, vol. 27, no. 1-2, pp. 79-88, 2002.

[7] W. A. Hare, E. WoldeMussie, R. N. Weinreb et al., "Efficacy and safety of memantine treatment for reduction of changes associated with experimental glaucoma in monkey, II: structural measures," Investigative Ophthalmology \& Visual Science, vol. 45, no. 8, pp. $2640-2651,2004$.

[8] E. WoldeMussie, E. Yoles, M. Schwartz, G. Ruiz, and L. A. Wheeler, "Neuroprotective effect of memantine in different retinal injury models in rats," Journal of Glaucoma, vol. 11, no. 6, pp. 474-480, 2002.

[9] J. Chen, Y. Miao, X. H. Wang, and Z. Wang, "Elevation of pNR2AS1232 by Cdk5/p35 contributes to retinal ganglion cell apoptosis in a rat experimental glaucoma model," Neurobiology of Disease, vol. 43, no. 2, pp. 455-464, 2011.

[10] M. G. Anderson, R. S. Smith, N. L. Hawes et al., "Mutations in genes encoding melanosomal proteins cause pigmentary glaucoma in DBA/2J mice," Nature Genetics, vol. 30, no. 1, pp. $81-85,2002$.

[11] S. W. M. John, R. S. Smith, O. V. Savinova et al., "Essential iris atrophy, pigment dispersion, and glaucoma in DBA/2J mice," Investigative Ophthalmology \& Visual Science, vol. 39, no. 6, pp. 951-962, 1998.

[12] R. T. Libby, M. G. Anderson, I. H. Pang et al., "Inherited glaucoma in DBA/2J mice: pertinent disease features for studying the neurodegeneration," Visual Neuroscience, vol. 22, no. 5, pp. 637-648, 2005. 
[13] C. L. Schlamp, Y. Li, J. A. Dietz, K. T. Janssen, and R. W. Nickells, "Progressive ganglion cell loss and optic nerve degeneration in DBA/2J mice is variable and asymmetric," BMC Neuroscience, vol. 7, article 66, 2006.

[14] B. P. Buckingham, D. M. Inman, W. Lambert et al., "Progressive ganglion cell degeneration precedes neuronal loss in a mouse model of glaucoma," The Journal of Neuroscience, vol. 28, no. 11, pp. 2735-2744, 2008.

[15] F. Schuettauf, K. Quinto, R. Naskar, and D. Zurakowski, "Effects of anti-glaucoma medications on gangion cell survival: the DBA/2J mouse model," Vision Research, vol. 42, no. 20, pp. 2333-2337, 2002.

[16] F. Schuettauf, R. Rejdak, M. Walski et al., "Retinal neurodegeneration in the DBA/2J mouse-a model for ocular hypertension," Acta Neuropathologica, vol. 107, no. 4, pp. 352-358, 2004.

[17] D. Reichstein, L. Ren, T. Filippopoulos, T. Mittag, and J. Danias, "Apoptotic retinal ganglion cell death in the DBA/2 mouse model of glaucoma," Experimental Eye Research, vol. 84, no. 1, pp. 13-21, 2007.

[18] W. K. Ju, K. Y. Kim, M. Angert et al., "Memantine blocks mitochondrial OPA1 and cytochrome c release and subsequent apoptotic cell death in glaucomatous retina," Investigative Ophthalmology \& Visual Science, vol. 50, no. 2, pp. 707-716, 2009.

[19] F. Schuettauf, S. Thaler, S. Bolz et al., "Alterations of amino acids and glutamate transport in the $\mathrm{DBA} / 2 \mathrm{~J}$ mouse retina; possible clues to degeneration," Graefe's Archive for Clinical and Experimental Ophthalmology, vol. 245, no. 8, pp. 1157-1168, 2007.

[20] M. Ji, Y. Miao, L. D. Dong et al., "Group I mGluR-mediated inhibition of Kir channels contributes to retinal muller cell gliosis in a rat chronic ocular hypertension model," The Journal of Neuroscience, vol. 32, no. 37, pp. 12744-12755, 2012.

[21] X. G. Luo, K. Chiu, F. H. S. Lau, V. W. H. Lee, K. K. L. Yung, and K. F. So, "The selective vulnerability of retinal ganglion cells in rat chronic ocular hypertension model at early phase," Cellular and Molecular Neurobiology, vol. 29, no. 8, pp. 1143-1151, 2009.

[22] R. Naskar, C. K. Vorwerk, and E. B. Dreyer, "Concurrent downregulation of a glutamate transporter and receptor in glaucoma," Investigative Ophthalmology \& Visual Science, vol. 41, no. 7, pp. 1940-1944, 2000.

[23] S. T. Li and J. G. Ju, "Functional roles of synaptic and extrasynaptic NMDA receptors in physiological and pathological neuronal activities," Current Drug Targets, vol. 13, no. 2, pp. 207-221, 2012.

[24] G. E. Hardingham, Y. Fukunaga, and H. Bading, "Extrasynaptic NMDARs oppose synaptic NMDARs by triggering CREB shutoff and cell death pathways," Nature Neuroscience, vol. 5, no. 5, pp. 405-414, 2002.

[25] A. M. Kaufman, A. J. Milnerwood, M. D. Sepers et al., "Opposing roles of synaptic and extrasynaptic NMDA receptor signaling in cocultured striatal and cortical neurons," The Journal of Neuroscience, vol. 32, no. 12, pp. 3992-4003, 2012.

[26] G. E. Hardingham and H. Bading, "Synaptic versus extrasynaptic NMDA receptor signalling: implications for neurodegenerative disorders," Nature Reviews Neuroscience, vol. 11, no. 10, pp. 682-696, 2010.

[27] S. Li, M. Jin, T. Koeglsperger, N. E. Shepardson, G. M. Shankar, and D. J. Selkoe, "Soluble A $\beta$ oligomers inhibit long-term potentiation through a mechanism involving excessive activation of extrasynaptic NR2B-containing NMDA receptors," The Journal of Neuroscience, vol. 31, no. 18, pp. 6627-6638, 2011.

[28] R. Rönicke, M. Mikhaylova, S. Rönicke et al., "Early neuronal dysfunction by amyloid $\beta$ oligomers depends on activation of NR2B-containing NMDA receptors," Neurobiology of Aging, vol. 32, no. 12, pp. 2219-2228, 2011.

[29] C. Tackenberg, S. Grinschgl, A. Trutzel et al., "NMDA receptor subunit composition determines beta-amyloid-induced neurodegeneration and synaptic loss," Cell Death and Disease, vol. 4, p. e608, 2013.

[30] A. Frasca, M. Aalbers, F. Frigerio et al., "Misplaced NMDA receptors in epileptogenesis contribute to excitotoxicity," Neurobiology of Disease, vol. 43, no. 2, pp. 507-515, 2011.

[31] J. Zhang and J. S. Diamond, "Subunit- and pathway-specific localization of NMD a receptors and scaffolding proteins at ganglion cell synapses in rat retina," The Journal of Neuroscience, vol. 29, no. 13, pp. 4274-4286, 2009.

[32] S. Zhang, L. Edelmann, J. Liu, J. E. Crandall, and M. A. Morabito, "Cdk5 regulates the phosphorylation of tyrosine 1472 NR2B and the surface expression of NMDA receptors," The Journal of Neuroscience, vol. 28, no. 2, pp. 415-424, 2008. 

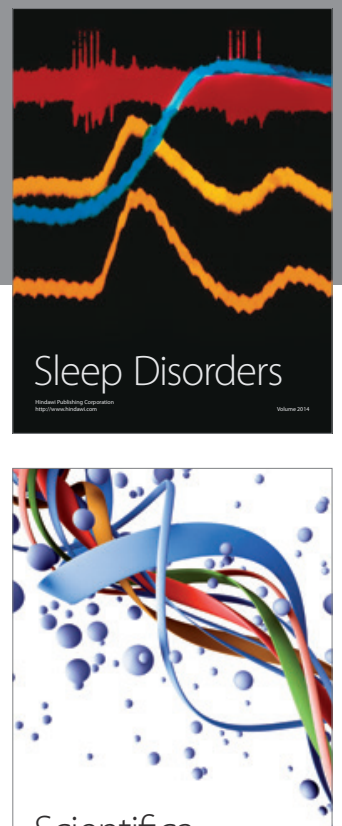

Scientifica

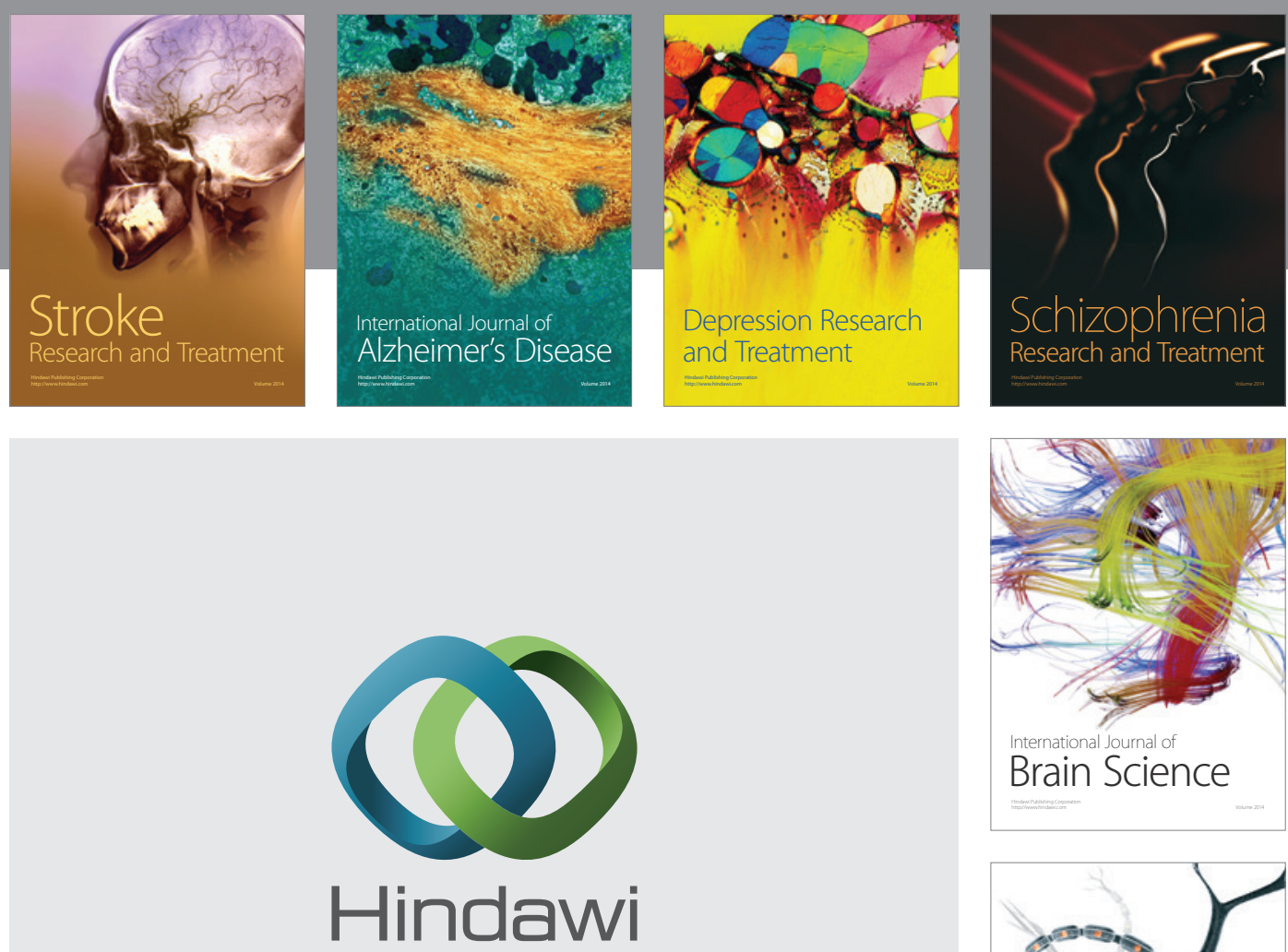

Submit your manuscripts at

http://www.hindawi.com
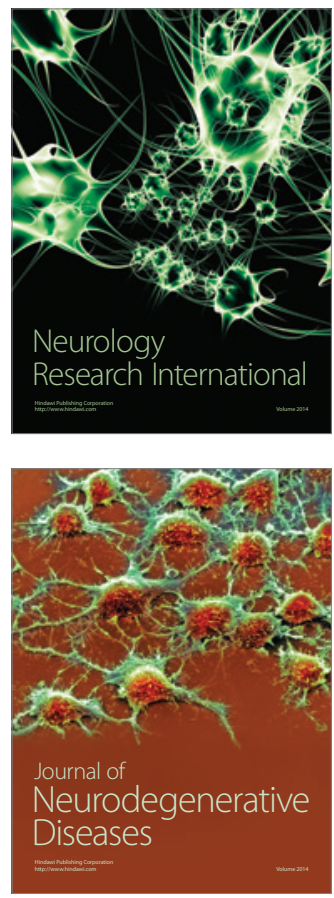

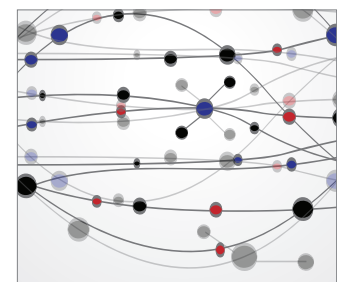

The Scientific World Journal
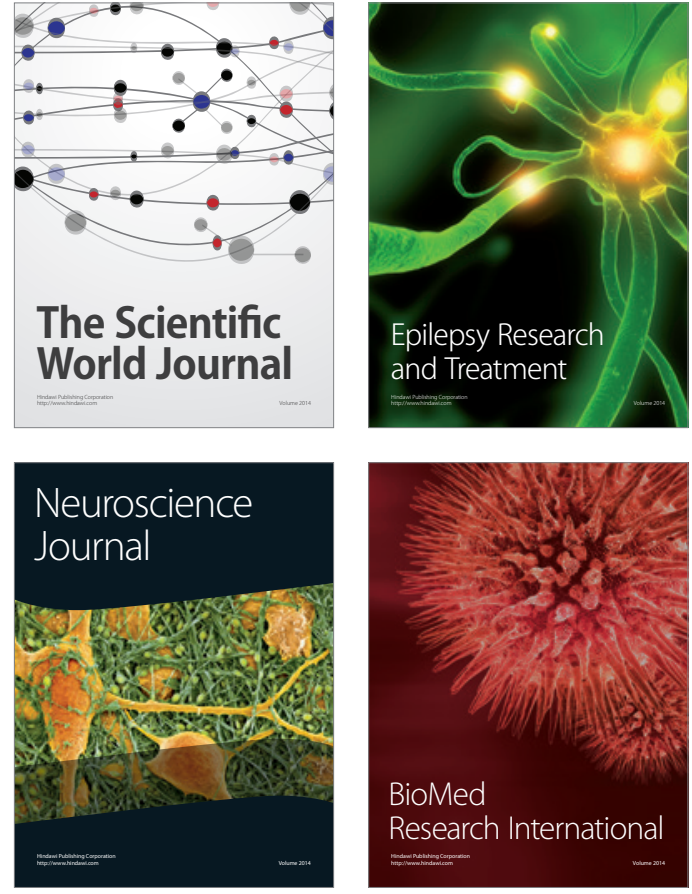



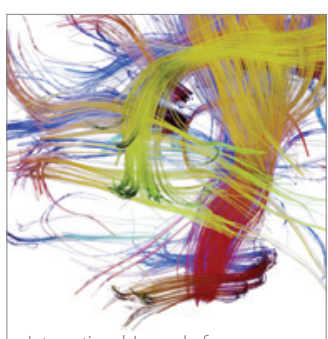

Brain Science

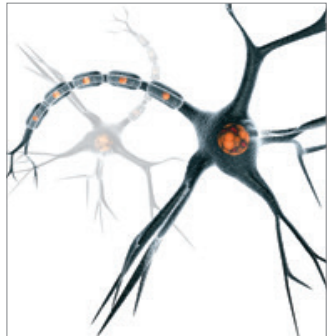

Neural Plasticity
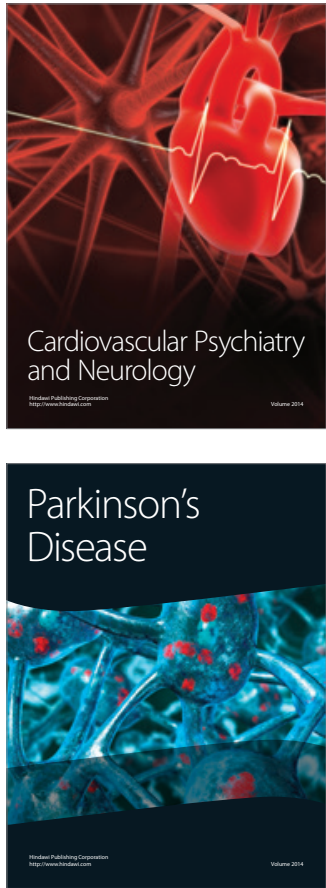